\title{
Capacity, Delay and Mobility in Wireless Ad-Hoc Networks
}

\author{
Nikhil Bansal
}

\author{
Zhen Liu
}

\begin{abstract}
Network throughput and packet delay are two important parameters in the design and the evaluation of routing protocols for ad-hoc networks. While mobility has been shown to increase the capacity of a network, it is not clear whether the delay can be kept low without trading off the throughput. We consider a theoretical framework and propose a routing algorithm which exploits the patterns in the mobility of nodes to provide guarantees on the delay. Moreover, the throughput achieved by the algorithm is only a poly-logarithmic factor off from the optimal. The algorithm itself is fairly simple. In order to analyze its feasibility and the performance guarantee, we used various techniques of probabilistic analysis of algorithms. The approach taken in this paper could be applied to the analyses of some other routing algorithms for mobile ad hoc networks proposed in the literature.
\end{abstract}

Keywords: Wireless Communication, Ad-Hoc Network, Network Capacity, Throughput, Mobility, Delay.

\section{INTRODUCTION}

We consider the problem of routing in ad-hoc wireless networks. Such a network is comprised of possibly mobile wireless nodes which attempt to communicate with each other in the absence of any fixed communication infrastructure. This ability of ad-hoc networks to form "networks on the fly" is expected to play a crucial role in situations such as disaster recovery efforts after an earthquake or fire, gathering information in a battlefield or simply forming a short-lived network among people attending a business meeting.

Routing efficiently in wireless ad-hoc networks poses many challenges. Some commonly studied problems are: How to handle the frequent changes in the network topology due to mobility of the users, failure of wireless links caused by obstruction or fading of signals. How to maintain the long multi-hop paths between two communicating nodes [7], [12], [13], [15], [14]. How to reduce the interference among the various users wishing to transmit which is caused due absence of any centralized control [20], [17], [19], [16], [18].

Another direction in the quest for efficient routing protocols was introduced by the work of Gupta and Kumar [4] which focused on the capacity of wireless ad hoc networks. They first show an upper bound on the maximum possible transmission capacity achievable by any static ad-hoc wireless network and then illustrate a routing protocol for a random network which has capacity close to the optimum. Such a result, though it ignores a lots of issues which arise in practical settings, offers important theoretical insights into the problem.

N. Bansal is with Computer Science Department, Carnegie Mellon University, Pittsburgh, PA 15213, USA. E-mail: nikhil@cs.cmu.edu. The Work was carried out while this author was working at IBM T. J. Watson Research Center.

Z. Liu is with IBM T. J. Watson Research Center, P. O. Box 704, Yorktown Heights, NY 10598, USA. E-mail: zhenl@us.ibm.com
Gupta and Kumar [4] show that the average available throughput per node decreases as the square root of the number of nodes $n$, in a static ad-hoc network. Equivalently, the total network capacity increases as at most $\sqrt{n}$. Their result holds quite generally. In particular, it holds irrespective of the network topology, power control policy or any transmission scheduling strategy.

Given this limitation on the achievable throughput, a natural question which arises is whether the average throughput available per node can be increased. There are two approaches discussed in the literature.

1) Add relay-only nodes in the network: This increases the total network capacity, thus increasing the share available to each sender [4]. However a major drawback of this scheme is that the number of relay nodes required is huge. For example, in a network with 100 senders, at least 4476 relay nodes are needed to increase the capacity five-fold [4].

2) Add mobility: In a network where nodes move randomly in a circular disk such that their steady state distribution is uniform, Grossglauser and Tse [2] showed that it is possible for each sender-receiver pair to obtain a constant fraction of the total available bandwidth. This constant remains independent of the number of sender-receiver pairs.

However, as noted in [2] their scheme does not provide any guarantee on the time that it takes for the packet to reach its destination, or on the size of the buffers needed at the intermediate relay nodes. In general, the delay to deliver the packet could be arbitrarily large. ${ }^{1}$

In this paper, we address the issue of whether mobility can be used in a way that not only can we obtain a close-to-optimal throughput as in [2] but also have low delay guarantees simultaneously. We consider a network with both static and mobile nodes. The mobile nodes follow a particular model of movement, referred to as the mobility model (see Section II). Our mobility model is similar to the random way-point model which is widely used in simulations of ad-hoc networks. We give a routing algorithm and prove that it provides high throughput and low delay.

We stress however that our work and similarly the results in [4], [2] are theoretical in nature. The various simplifying assumptions used about the network conditions and scenarios need not always hold in real world settings. For example, one assumption often made in obtaining lower bounds on the throughput is that all the nodes are distributed uniformly at random in the network. Similarly, the sender receiver pairs are also

\footnotetext{
${ }^{1}$ Later we will look at these schemes in more detail.
} 
chosen randomly. Indeed, if all nodes wish to send packets to one receiver, no node can receive more than $1 / n^{\text {th }}$ of the bandwidth, where $n$ is the number of the nodes. Similarly, if most nodes are concentrated in a small region, only one of them can communicate at once due to excessive interference. All our assumptions are explicitly stated in section II-F.

The paper is organized as follows. In Section II-A through II-F, we define our model and state the modeling assumptions. Section II-E contains a discussion of the previous algorithms and gives intuition for the problem. Section II-G states our main result. Section III describes the routing algorithm and its analysis. In Section IV we consider some extensions of the model. Finally we end with discussions and conclusions in Sections V and VI.

\section{Model And Main Results}

\section{A. Network Model}

The ad-hoc network consists of $n$ static nodes and $m$ mobile nodes all lying in a disk of unit area (of radius $1 / \sqrt{\pi}$ ). The location of the static nodes are fixed. The static nodes are distributed uniformly at random over the unit circular disk. The mobile nodes are randomly distributed in the disk at time $t=0$. At later times their position and velocities are given by the mobility model described below.

\section{B. Mobility models}

Various mobility models have been considered in the literature to evaluate the effect of the node mobility on the performance of algorithms and protocols. The most widely used of these is probably the "random waypoint model" [7] (described below).

Random waypoint Model: In this model, a node chooses a destination distributed uniformly at random in the unit disk and moves in that direction with a speed $v$. The speed is chosen uniformly in some interval $\left(0, v_{\max }\right]$. On reaching the destination the node pauses for some time distributed according to some random variable and the process repeats itself.

Other models include Random Gauss-Markov [9] and Fluid flow models [22]. A nice survey of these can be found in [6], [9].

In this paper, we define the Uniform Mobility Model. This model has the advantage of being analytically tractable and is similar to the "random waypoint model".

Uniform Mobility Model: In this model, each of the $m$ mobile nodes move at speed $v$ inside the unit circular disk. At time $t=0$, the position of these nodes are distributed uniformly at random inside the disk. Moreover, the directions of motion of the $m$ nodes at time $t=0$ are i.i.d. and uniformly distributed in $[0,2 \pi)$.

At subsequent times a node behaves as follows: It picks a direction uniformly at random from $(0,2 \pi]$ and moves in that direction for a distance $d$, at speed $v$, where $d$ is an exponentially distributed random variable with mean $\mu$. The process repeats when the node reaches the distance $d$. If the node hits the boundary of the disk, it is reflected at the boundary.

The uniform mobility model satisfies the following properties given by Theorem 1 below, the proof of which is forwarded to Appendix VII-B.
Theorem 1 The Uniform mobility model described above has the following properties:

1) Given a time $t$, the position of the mobile nodes at time $t$ are independent of each other.

2) The steady state distribution of the mobile nodes over the disk is uniform.

3) Conditional on the position of a mobile node in the disk, the direction of the node is uniformly distributed in $(0,2 \pi]$.

\section{Transmission Model}

At time $t$, let $S_{1}, S_{2}, \ldots, S_{m}$ be the senders with positions $X_{1}, \ldots, X_{m}$ and let $R$ be the receiver with position $X_{0}$. If $S_{i}$ uses power $P_{i}(t)$ for transmission, the strength of the signal received at $R$ is $\frac{P_{i}(t)}{\left\|X_{i}-X_{0}\right\|^{\alpha}}$, where $\alpha>2$. The transmission from $S_{i}$ to $R$ is successful if

$$
\frac{P_{i}(t)\left\|X_{i}-X_{0}\right\|^{-\alpha}}{N+\sum_{k \neq i} P_{k}(t)\left\|X_{k}-x_{0}\right\|^{-\alpha}} \geq \beta
$$

The constant $\beta$ is the Signal-to-Noise Ratio (SNR) for the transmission channel.

The maximum available bandwidth to any node in the network will be denoted by $W$.

\section{The Performance Metric}

We will use the framework of [4] and [2]. There will be $n$ sender-destination pairs. In particular, each static node $i$ will act as a source (aka sender) transmitting to some other static destination (aka receiver) node $d(i)$. Each source chooses its destination uniformly at random (as in [2], [4]).

A scheduling policy $\gamma$ chooses which sender sends data at time $t$, and the power levels $P_{i}(t)$ for node $i$. Given a scheduling and relay policy $\gamma$, we will say that $\gamma$ achieves a throughput of $\lambda(n)$ if it is possible for each source to send data at the rate $\lambda(n)$.

\section{E. Preliminaries}

Before giving our results, we first give an intuitive explanation of results known previously in the literature. We will also make some observations which will motivate our problem further and illustrate the main ideas.

Intuitively, if a node $S$ transmits a message to some node at a distance, $d$, then due to the nature of wireless transmission, this causes an interference to all the nodes within a distance of approximately $d$ from $S$. Hence, if the average distance of transmission is about $d$, then at most $n / d^{2}$ users can transmit simultaneously.

This forms the basis of the result of Gupta et al [4]. If a node transmits a packet to another node $d$ steps away, then in a disk topology, the number of hops between source to destination will be $\sqrt{n} / d$ on the average. This implies that the total throughput can be at most $\left(n / d^{2}\right) /(\sqrt{n} / d)=\sqrt{n} / d$. Thus, it helps to have short range transmissions (i.e. $d=1$ ) and hence the total capacity can increase at most as $\sqrt{n}$.

Obtaining an $\Omega(1)$ average throughput per node is a very stringent requirement and it implies several things. First, this 
means that each node must be sending packets to its destination for a constant fraction of the time. Second, each packet traveling from source to destination must involve at most a constant number of relays. The idea of Grossglauser et al [2] is that each node hands over a packet to its nearby mobile node at all times. When the mobile node is close to the destination node, it hands over the packet to the destination. Note that this does not provide any guarantees on how long the packet will take to reach the destination [2].

In our scenario we need to ensure a bounded delay and a good throughput simultaneously. To do this we need to ensure several things.

1) To provide good delay guarantees we need to assume that the position of the destination is fixed. Indeed, if the destination is a mobile node, it will be impossible to provide any guarantees on the delay, unless some assumptions are made (See Section IV-A). Hence we will assume that the sources and destinations are static nodes, which use the mobile nodes as relays in order to achieve a throughput greater than $\sqrt{n}$.

2) Second, to obtain a constant throughput per sender, senders need to be able to transmit most of the time. This requires that number of mobile nodes must be at least $\Omega(n)$, since otherwise the throughput is bounded by the number of static nodes $(n)$.

Similarly, if the number of mobile nodes is $o(\sqrt{n})$, then at any time at most $o(\sqrt{n})$ static nodes could be communicating with mobile nodes and thus the throughout will only be $o(\sqrt{n})$. Hence, it suffices to use the algorithm of Gupta et al in this case. Infact, throughout this paper we will assume that the number of mobile nodes, $m$, is a number between $\sqrt{n}$ and $n$.

3) The number of relays per packet should not be too large. To do this we will need to exploit the patterns in the mobility of nodes.

4) Finally, to ensure a small delay, we must ensure that a packet does not get strayed along the path. This requires new ideas and we do not know of any previous work which considers these issues. Note that, at any time, a relay node will have several packets corresponding to various destinations. However, when it meets another relay node along its way, it can hand over very few of these packets, since the duration during which they are nearest neighbors (hence are in communicating range) is quite small. Hence we need a scheme to decide which packets need to be handed off. Observe that this is not a issue if delays are not taken into consideration. Handling and analyzing delays is perhaps one of the most important contributions of this paper.

Lastly, we note that another possible model could be where the mobile nodes are dedicated as relays and their paths and motion are dictated by the requirements of the static nodes. However, this gives a different feel to the problem, in this case it can be modeled as a network flow problem. In our model, the mobile nodes correspond to users traveling in cars, airplanes etc., which have their own pattern of movement. These users have a wireless device, which potentially other static devices can use. But clearly, we cannot expect the wireless device to dictate the movement of the user.

\section{F. Modeling Assumptions}

We will assume that the following hold:

1) The locations of the static nodes are known to other nodes. This is a reasonable assumption since this information can be obtained through some location services or protocols (see e.g. [8]) and then stored in the nodes.

2) The mobile nodes know the direction in which they are moving up to some degree of accuracy. This is easy to do if the network has Global positioning system (GPS) support. Even if GPS support is unavailable, the mobile nodes can detect its direction by observing the sequence of static nodes which are closest to it which it encounters. Detecting close-by static nodes is commonly done using signal strengths.

3) A mobile node knows how far it will move in a line before changing direction. This for example is true in the random way point model, and also in real life situations where nodes know their destination.

4) We assume for ease of exposition that $\mu$ (the parameter in the mobility model) is of the order of the diameter of the network. In Section IV we remove this assumption.

\section{G. Main Results}

Our main result is the following.

Theorem 2 Consider a mobile ad hoc network satisfying the following conditions:

1) There are $n$ static nodes uniformly distributed in a disk, and $m$ mobile nodes which move according to the uniform mobility model in the unit disk.

2) The $n$ sender-receiver pairs are chosen randomly according to a uniform distribution, among the static nodes.

Then, there exists a constant $c>0$, such that each sender can achieve an average throughput of $c \frac{W \min (m, n)}{n \log ^{3} n}$, where $W$ is the maximum available bandwidth. Moreover, the maximum delay incurred by the packet is at most $\frac{2 d}{v}$, where $d$ is the diameter of the network and $v$ is the velocity of the mobile nodes.

Note that the best achievable throughput by any algorithm with $m$ mobile nodes is $O(W m / n)$, where Theorem 2 provides a guarantee of $\Omega\left(W m /\left(n \log ^{3} n\right)\right)$.

\section{Routing Algorithm}

We begin with a high level description of the routing algorithm. We then describe the details and the analysis. More general models will be analyzed in the next section.

\section{A. Algorithm}

Figure 1 shows the routing of a packet from the source $S$ to the destination $R$. The packet moves approximately in the direction of the destination at each step.

At a high level, the algorithm consists of the following steps: 


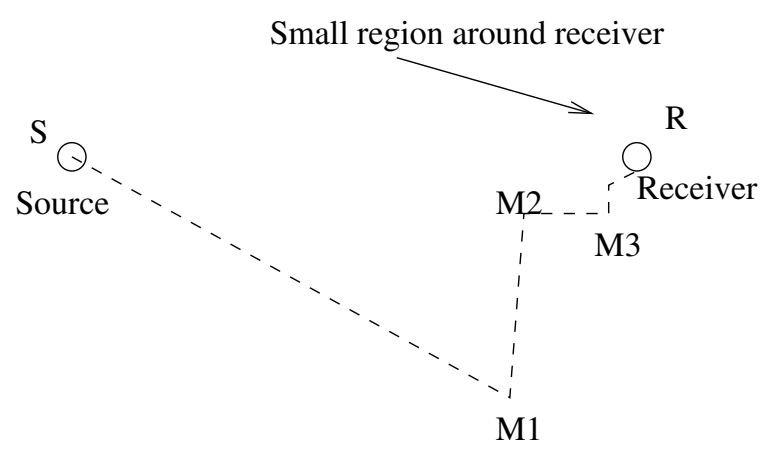

Fig. 1. An example illustrating the routing scheme

1) Within each region of size $1 / \sqrt{m} \times 1 / \sqrt{m}$, the static nodes choose a local leader. This leader will be responsible for communicating all the messages of the static nodes in its region with the mobile nodes. Note that there will be $m$ local leaders.

2) A static node $S_{1}$ wanting to send a message to destination $R$ first transfers its message to its leader node $(S)$. $S$ stores the message and waits for a mobile node $\left(M_{1}\right)$ such that $M_{1}$ is close enough to $S$ and moving approximately along the direction of $R$. When such a node is available, $S$ hands over the data to be transmitted to it. We will call this the Static-to-Mobile phase.

3) The mobile nodes relay the packets intended for $R$ amongst themselves such that the packet moves closer and closer to the destination. We will call this the Mobileto-Mobile phase.

4) When the mobile relay carrying the packet is close enough to the destination, it hands off the packet to some leader node. This packet is then routed among the leader nodes towards the correct leader node, which then transmits the packet to the destination node. This is the Staticto-Static phase.

Observe that in the algorithm, the various phases are executing simultaneously. The signals used in these different phases will cause interference to each other. In our analysis, we will assume that each of these phases use different frequency channels, and hence they do not interfere with each other. In particular, we will assume that each of the phases has a maximum allocated bandwidth of $W / 4$. In the entire algorithm, we will assume that all nodes transmit at unit power. The proof idea will be to show that each leader node can obtain a throughput of $\Omega\left(W / \log ^{3} n\right)$. Since within each region of a leader, there are $O(n / m)$ static nodes. This implies that leader static node can get a throughput of $\Omega\left(W m / n \log ^{3} n\right)$. Next we consider each of these steps in detail. The most involved part of the proof will be the Mobile-to-Mobile phase (in Section III-D). We will need to argue several things: Most importantly, a scheme for deciding which of the several data packets to handoff when two mobile nodes meet. Showing the feasibility of relaying the packets from the source to a node close enough to the destination. Making high probability arguments about packet loss, achievable throughput etc.

\section{B. Choosing a local leader}

We first describe how to choose a local leader. If $m>$ $n /(16 \log n)$, let $m=n /(16 \log n)$. Consider a virtual grid consisting of $m$ nodes with the distance between the neighbors being $m^{-\frac{1}{2}}$. It follows from a standard Chernoff bound argument (Appendix VII-A) that each of the grid nodes has a static node within a distance of $\sqrt{\log n / n}$, with probability at least $1-n^{-2}$. We choose one of these static nodes as the leader node corresponding to the square region of side $m^{-\frac{1}{2}}$ around each grid node. Since the grid nodes are separated by a distance of $m^{-\frac{1}{2}}$, the distance between any two leader nodes will be at least $m^{-\frac{1}{2}}-2 \sqrt{\log n / n}$. Finally, applying Chernoff bounds again, we observe that each region consists of $O(n / m)$ static nodes. Intuitively, if we ignore the static nodes that are not leaders, then the network has $m$ mobile nodes and $m$ leaders where the leaders are arranged in a grid like structure (i.e. no two are very close to each other).

The communication will take place as follows: In each region (cluster) only the leader node will be responsible for communicating with the mobile node. A static node that wants to transmit a packet will first send the packet to the leader of the cluster to which the static node belongs. The packet will then be routed among the leader and the mobile nodes. Finally, the leader sends the packet to the destination by local routing within a cluster. Note that if the throughput available to the leader is $\lambda$, then each static node gets throughput of $\Omega(m \lambda / n)$. For example, the static nodes corresponding to a particular leader can take turns transmitting packets to the leader.

\section{Static-to-Mobile Phase}

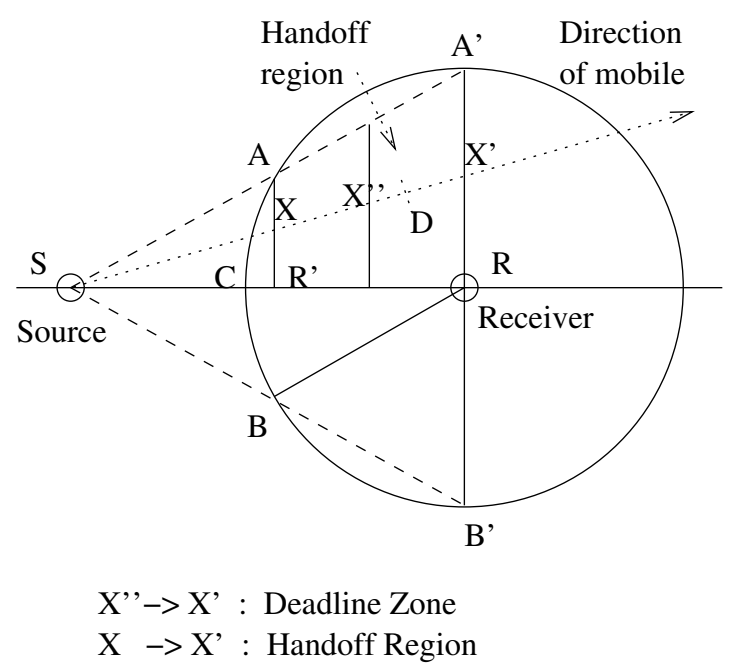

Fig. 2. Handing off the packet to the mobile node

In this mode, a static node first hands off the data to be delivered to a mobile node. Consider Figure 2, given the source node $S$ (which wants to handoff data to another mobile node) and the destination node $R$. Consider the line joining $S$ and $R$, let $\theta$ be the slope of $S R(\theta=0$ in Fig. 2). Let $G(S, R)$ denote the region between the directions $\left[\theta-\frac{\pi}{6}, \theta+\frac{\pi}{6}\right]$ originating at $S$ and containing $R$ (The choice of the angle $\pi / 6$ is arbitrary, any angle from 0 to $\pi / 3$ would suffice, see Remark 2 at the end of 
Section III-D.1) . In Figure 2 the region $G(S, R)$ corresponds to the region between the rays $S A^{\prime}$ and $S B^{\prime}$.

In the first step, the node at $S$ hands over the packet to be transmitted to some mobile node $M_{1}$ moving in the direction $\phi$ such that $\phi \in\left[\theta-\frac{\pi}{6}, \theta+\frac{\pi}{6}\right]$, also $M_{1}$ satisfies the property that it moves in that direction for a distance of at least $2|S R|$ (i.e. twice the length of segment SR). Since $\mu$ is about the diameter of the network, by our assumption, a constant fraction of the mobile nodes satisfy this property. We will assume that this is always the case in all transmissions. We make this assumption to simply the exposition, so that the main ideas are not obscured. In Section IV we get rid of this assumption.

There are two issues that arise in this phase:

1) Whether a static node transmitting at a rate $\lambda(n)$ can find mobile nodes moving in the right direction sufficiently often to handoff data at that rate.

2) How long does a node have to wait until it can find the right mobile node.

The next two results consider these issues.

Theorem 3 If the communication takes place only between leader nodes as senders and mobile nodes as receivers. Then, if $\alpha>2$, there is a square region of side $\mathrm{cm}^{-\frac{1}{2}}$ centered at each leader, such that if the leader communicates with a mobile node in this region, then the communication is always a success.

Proof: Consider the static node $(S)$ at $(0,0)$. Let $c$ be a sufficiently small constant such that $c<1$. If a mobile node $(M)$ is present inside the square of size $d=\mathrm{cm}^{-\frac{1}{2}}$, then the distance of $M$ from $S$ is at most $\frac{d}{\sqrt{2}}$. Now, since nodes transmit at unit power, the signal received by $M$ due to $S$ will be at least $2^{\frac{\alpha}{2}} m^{\frac{\alpha}{2}} c^{-\alpha}$.

To bound the interference by other nodes, note that there are $8 l$ nodes at positions $(i, j)$ such that $\max (i, j)=l$. Each of these $8 l$ nodes have distance at least $l m^{\frac{1}{2}}-\frac{d}{2}$ from $M$ which is $\geq\left(l-\frac{1}{2}\right) m^{-\frac{1}{2}}$, as $c<1$.

Thus the total signal at $S$ due to the other nodes is

$$
\begin{aligned}
& \leq \sum_{l=1}^{\infty} \frac{8 l}{\left(l-\frac{1}{2}\right)^{\alpha} m^{-\frac{\alpha}{2}}} \\
& \leq \frac{8 m^{\frac{\alpha}{2}}}{\left(\frac{1}{2}\right)^{\alpha}}+8 m^{\frac{\alpha}{2}} \int_{1}^{\infty} \frac{x}{\left(x-\frac{1}{2}\right)^{\frac{\alpha}{2}}} d x \\
& =8 m^{\frac{\alpha}{2}} 2^{\frac{\alpha}{2}}+8 m^{\frac{\alpha}{2}}\left[\frac{2^{\alpha-2}}{\alpha-2}+\frac{2^{\alpha-1}}{\alpha-1}\right] \\
& =c_{1} m^{\frac{\alpha}{2}}
\end{aligned}
$$

Equation (3) follows from Equation (2) since $\frac{x}{\left(x-\frac{1}{2}\right)^{\frac{\alpha}{2}}}$ is a decreasing function of $x$ for $x>1 / 2$ and $\alpha>2$.

Thus the signal to noise ratio at $M$ due to $S$ will be greater than or equal to

$$
\frac{2^{\frac{\alpha}{2}} m^{\frac{\alpha}{2}} c^{-\alpha} / N}{1+c_{1} m^{\frac{\alpha}{2}} / N}
$$

Thus given the Signal to Noise Ratio $\beta, c$ can be chosen appropriately small such that the signal to ratio given by Equation (4) is greater than $\beta$. Hence the transmission from $S$ to $M$ is always successful.
For a static node $S$, let $N(s)$ denote the square neighborhood of side $\mathrm{cm}^{-\frac{1}{2}}$ centered at $S$. We will now show that the duration for which a static node $S$ has to wait to find a mobile node in $N(s)$ is not very high.

Lemma 4 Consider a static node $S$. Let $T$ denote the time until which a mobile node first enters $N(s)$. Then

$$
\operatorname{Pr}\left[T>\frac{4 f \log m}{c v} \sqrt{\frac{1}{m}}\right] \leq m^{-f}
$$

Proof: Let $M$ be some mobile node at a distance $r$ from $S$. Now the direction of motion of $M$ is uniformly distributed and independent of its position. Conditioned on the fact that $M$ does not change direction, the probability that $M$ enters the neighborhood $N(s)$ of the static is equal to the angle subtended by the region $N(s)$ at $M$. This subtended angle is at least $\frac{c}{\sqrt{m}}$.

Consider concentric rings $D_{1}, \ldots, D_{i}, \ldots$ of width $\frac{1}{\sqrt{m}}$ defined as follows. Each $D_{i}$ is centered at $S$ and $D_{i}$ consists of points which are at a distance between $\frac{i-1}{\sqrt{m}}$ and $\frac{i}{\sqrt{m}}$ from $\mathrm{S}$.

Let $X_{i, j}$ be a random variable such that $X_{i, j}=1$ if the $i^{\text {th }}$ mobile node is in $D_{j}$ and its direction is such that it will enter $N(s) . X_{i, j}$ is 0 otherwise. Since the mobile nodes are uniformly distributed in the unit disk, the probability that $M_{i} \in D_{j}$ is $\left(j^{2}-\left(j-\frac{1}{\sqrt{m}}\right)^{2}\right)$ which is approximately $2 j m^{-\frac{1}{2}}$. Since each mobile in $D_{j}$ has a probability of at least $\frac{c}{j \sqrt{m}}$ of entering $N(s)$. The event $X_{i, j}$ has an expected value of $2 j m^{-\frac{1}{2}} \frac{c}{j \sqrt{m}}=\frac{2 c}{m}$. Thus $X_{i, j}$ are independent Bernoulli random variables. Define $X(d)=\sum_{j=1}^{d} \sum_{i=1}^{m} X_{i, j}$. Observe that $E[X(d)] \geq 2 c d$.

We are interested in obtaining a bound on $d$ such that $X(d) \geq$ 1. If $d>1 / c, \operatorname{Pr}[X(d)<1] \leq \operatorname{Pr}[X(d)<c d]$.

Using the second Chernoff bound Equation (10) with $\delta=\frac{1}{2}$, we get $\operatorname{Pr}[X(d)<c d]<e^{-\frac{2 c d}{8}}$.

Thus probability that $X(d)<1$ for $d=4 f \log m / c$ is less than $m^{-f}$. Now, observing that the time taken for a mobile to reach $S$ from a distance $x$ is at most $x / v$ the result follows.

\section{Mobile-to-Mobile Phase}

We now describe and analyze in detail how handoffs from one mobile node to another take place. The main ideas are the following: First, for every data packet carried by a mobile node there is a point beyond which the packet is not handed to another mobile node, it starts moving away from the destination. This leads to the notion of a handoff region. We have to make sure that every packet is handed off to another mobile node within its handoff region. Second, for every mobile node, at any moment in time there will be several packets in their handoff region. We thus need a scheme to decide which packet should be handed off first and so on. The second scheme also requires the use of randomization so that delay guarantees can be proven. These two ideas are described immediately below and Section III-D. 2 contains the analysis of these.

1) Handoff algorithm: Geometry: Consider Figure 2, the mobile node $M_{1}$ was handed a packet from the static node $S$. Now, $M_{1}$ carries the packet until it reaches a region between the rays $R^{\prime} A$ and $R A^{\prime}$. In this region, $M_{1}$ tries to hand off the 
packet to another mobile node $M_{2}$, which is moving towards $R$. This region will be denoted as the handoff region. The handoff region is defined as follows. Line RA' is perpendicular to line $S R$. Line $R^{\prime} A$ is parallel to line $A^{\prime} R$ and passes through $A$. The handoff region is shown by the segment $X X^{\prime}$ in Figure 2.

Scheduling: In order to handle packets that could not be transmitted in their handoff regions we will define a deadline. Let $X^{\prime \prime}$ denote the midpoint of the segment $X X^{\prime}$. The mobile node chooses a point uniformly at random in the segment $X^{\prime \prime} X^{\prime}$. This point is called the deadline for the node ${ }^{2}$. The deadline is shown by $D$ in Figure 2 . If the mobile node $M_{1}$ is unable to handoff the packet to another mobile until it reaches the deadline $D$ (i.e. during the interval $X$ to $D$ ), the packet is discarded.

Finally, note that at any given time several packets will be in their handoff regions, and hence compete for being handed off to another mobile node. The conflict resolution among the various packets is done in the Earliest Deadline First (EDF) order. For example if either of the packets $P_{1}$ with deadline $D_{1}$ and packet $P_{2}$ with deadline $D_{2}$ can be delivered to a mobile node $M_{2}$, then the packet with the earlier deadline is delivered first.

The whole process repeats itself, until the mobile reaches a distance of less than $c \frac{\log m}{\sqrt{m}}$ from the destination. The appropriate constant $c$ will be determined later.

Remarks:

1) Observe that the algorithm is fully distributed, in the sense that the communication between two mobile nodes does not impose any restriction on the transmissions from other mobile nodes.

2) Note that the choice of the angle $\pi / 6$ is arbitrary. In fact, a handoff to any mobile moving within an angle of $(0, \pi / 3]$ suffices, since at each step the distance to the destination decreases. The tradeoff is that a smaller choice of the angle implies a longer wait, whereas a bigger angle implies a lesser wait but a possibly longer path to reach the destination.

2) Analysis of the mobile-to-mobile phase: Call a handoff successful if the packet is delivered from a mobile $M_{i}$ to a mobile $M_{j}$ such that the packet was in the handoff mode at the time of delivery and it was delivered before the deadline. We first determine the conditions for a successful handoff.

Let $R$ be a receiving mobile node and let $M_{1}, \ldots, M_{m}$ be mobile nodes distributed uniformly and independently in the disk. Suppose that $M_{1}$ attempts to communicate with $R$. Then the signals due to $M_{2}, \ldots, M_{m}$ received at $R$ will cause interference to the communication between $M_{1}$ and $R$. Let $I_{j}$ denote the signal received at $R$ due to $M_{j}$. To determine the probability of successful transmission between $M_{1}$ and $R$, we will bound the total interference $I_{2}+\ldots+I_{m}$. This is done in the following lemma and its proof is similar to that in [5], [2]. However, we include it for completeness.

Lemma 5 Suppose that $m-1$ mobile nodes uniformly distributed in a disk are transmitting at unit power. Let $R$ be a

\footnotetext{
${ }^{2}$ Randomization is required to avoid many deadlines from being clustered together, its use will be apparent in the proofs below
}

point in the interior of the disk. Then, if $M$ is at a distance $x<\beta^{-\frac{1}{\alpha}} \sqrt{\frac{\epsilon}{\pi m}}$ to point $R$, the transmission from $M$ to $R$ is successful with probability greater than $1-\epsilon$. In particular, if $x<\beta^{-\frac{1}{\alpha}}(8 \pi m \log m)^{-\frac{1}{2}}$, then the transmission is a success with probability at least $\frac{1}{8 \log m}$.

Proof: Let $X(R)$ be the position of $R$. Consider a disk $D$ of radius $r=\pi^{\frac{1}{2}-|X(R)|}$ centered at $X(R)$. Then the disk $D$ lies entirely inside the unit disk. Then for all $z>r^{-\alpha}$ and for all $j$

$$
\operatorname{Pr}\left[I_{j} \geq z\right]=\pi z^{-\frac{2}{\alpha}}
$$

Observe that each of the $I_{j}^{\prime} s$ are distributed i.i.d. with the tail distribution given by Equation 5. Since $\alpha>2$, note that the $I_{j}^{\prime} s$ have a Pareto tail distribution with exponent less than 1.

To estimate $I_{2}+\ldots+I_{m}$, we use the limit theorem for sums of Pareto distribution with exponent less than 1 [1]. Let $\gamma=\frac{2}{\alpha}$. Observe that $[\pi \Gamma(1-\gamma)]^{-\frac{1}{\gamma}} I_{j}$ has the same tail distribution as $G_{\gamma}$, where $G_{\gamma}$ is the stable distribution with exponent $\gamma$, i.e.

$$
1-G_{\gamma}(x)=\frac{x^{-\gamma}}{\Gamma(1-\gamma)}
$$

Let $I(m-1)=I_{2}+I_{3}+\ldots+I_{m}$ and $S(m-1)=$ $[\pi \Gamma(1-\gamma)]^{-\frac{1}{\gamma}} I(m-1)$. Then by [1], as $m \rightarrow \infty$

$$
\lim _{m \rightarrow \infty} \frac{S(m-1)}{(m-1)^{\frac{1}{\gamma}}} \rightarrow G_{\gamma}
$$

Thus we get

$$
\begin{aligned}
& \lim _{m \rightarrow \infty} \operatorname{Pr}\left[S(m-1)>x m^{\frac{\alpha}{2}}\right]=\frac{x^{-\gamma}}{\Gamma(1-\gamma)} \\
& \text { Setting } x=(\epsilon \Gamma(1-\gamma))^{\frac{-1}{\gamma}}, \text { we get } \\
& \lim _{m \rightarrow \infty} \operatorname{Pr}\left[I(m-1)>\left(\frac{\pi}{\epsilon}\right)^{\frac{\alpha}{2}} m^{\frac{\alpha}{2}}\right] \leq \epsilon
\end{aligned}
$$

Setting $\epsilon=\frac{1}{8 \log m}$ in Equation 6 gives

$$
\lim _{m \rightarrow \infty} \operatorname{Pr}\left[I(m-1)>(8 \pi m \log m)^{\frac{\alpha}{2}}\right] \leq \frac{1}{8 \log m}
$$

Now observe that $I(m-1) \rightarrow \infty$ as $m \rightarrow \infty$. Thus while comparing the signal to noise ratio we can ignore the contribution of the thermal noise (as denoted by $N$ in Equation 1). Thus if the signal from $M_{1}$ is greater than $\beta I(m-1)$ the transmission is a success. Hence by Equation 7, the transmission is a success with probability at least $\frac{1}{8 \log m}$ if the distance of $M_{1}$ from $R$ is less than or equal to $\beta^{-\frac{1}{\alpha}} 8 \pi m \log m^{-\frac{1}{2}}$

Let $r_{0}$ denote the distance $\beta^{-\frac{1}{\alpha}}(8 \pi m \log m)^{-\frac{1}{2}}$. Suppose two mobile nodes $M_{1}$ and $M_{2}$ communicate only when they are at a distance of $r_{0}$ or less. We will say that $M_{1}$ and $M_{2}$ encounter each other within time $t$, if the distance between them is less than $\frac{r_{0}}{2}$ during some time instant in $\left[t_{0}, t_{0}+t\right]$, where $t_{0}$ denotes the current time. We will be interested in the amount of data $M_{1}$ can handoff to another mobile during an encounter. This amount will depend on the number of mobile nodes that $M_{1}$ encounters and the amount of data that can be transmitted per encounter. 
Lemma 6 Let $\lambda_{m m}$ denote the bandwidth allocated for mobile-to-mobile data transmission. Then,

1) The expected data transmitted successfully during an encounter is at least $\frac{\lambda_{m m} r_{0}}{2|| v||}(1-1 / 8 \log m)$.

2) Let $E$ denote the event that at least $\frac{\lambda_{m m} r_{0}}{4|| v||}$ data is successfully transmitted during an encounter. Then, $\operatorname{Pr}[E] \geq(1-1 / 4 \log m)$. Similarly, the probability that at least $\frac{k \lambda_{m m} r_{0}}{4|| v||}$ data is successfully transmitted during $k$ encounters, is at least $(1-1 / 4 \log m)$.

Proof: Consider the motion of $M_{2}$ relative to $M_{1}$. Then $M_{2}$ moves at speed at most $2\|v\|$. Moreover, during an encounter $M_{2}$ covers a distance of at least $r_{0}$ during which it is at a distance of $r_{0}$ or less from $M_{1}$. Thus during an encounter, $M_{1}$ and $M_{2}$ are within a distance of $r_{0}$ for a duration of at least $\frac{r_{0}}{2\|v\|}$. Hence, by Lemma 5 the expected amount of data communicated during an encounter is at least $\frac{\lambda_{m m} r_{0}}{2\|v\|}\left(1-\frac{1}{8 \log m}\right)$. Now, it follows from Markov's Inequality that the amount of data transmitted successfully is at least $\frac{\lambda_{m m} r_{0}}{4|v| \mid}$ with probability $\frac{1}{4 \log m}$. Similarly, using Markov, the result follows for $k$ encounters.

We now consider the number of encounters which $M_{1}$ has in time $t$. Suppose the mobile node $M_{1}$ is wants to transmit data to a destination in the direction $\eta$. Thus $M_{1}$ will handoff the packet to some other mobile node $M_{i}$ moving in the direction $\phi$, such that $\phi \in[\eta-\pi / 6, \eta+\pi / 6]$.

Given $M_{1}$, call a mobile node $M_{i}$ useful if:

1) $M_{i}$ moves in the direction $\phi$ such that $\phi \in\left[\eta-\frac{\pi}{6}, \eta+\frac{\pi}{6}\right]$

2) $M_{i}$ encounters $M_{1}$ at sometime during the time $\left[t_{0}, t_{0}+\right.$ $t]$.

Let $X(\eta, t)=\mid\left\{i \mid i>0\right.$ and $M_{i}$ is useful $\} \mid$

That is, $X(\eta, t)$ is the random variable, which denotes the number of useful mobile nodes.

We now compute the expectation of $X(\eta, t)$ and bound it as in Lemma 4 to obtain the desired result.

Lemma 7 Let $M_{0}$ be a mobile node and let $X(\eta, t)$ be as defined above. Then for any $\eta \in[0,2 \pi)$,

$$
E[X(\eta, t)]>\frac{1}{24} m r_{0} t\|v\|
$$

Moreover, for any constant $k>0$, if $t>\frac{\log m}{\sqrt{m}|| v||}$, then

$$
\operatorname{Pr}\left[X(\eta, t)<\frac{1}{2} \cdot \frac{1}{24} m r_{0} t\|v\|\right]=o\left(\frac{1}{\log m}\right)
$$

Proof: Clearly, $X(\eta, t)$ is a sum of $m-1$ i.i.d. Bernoulli random variables $Y_{i}$, where $Y_{i}=1$ if $M_{i}$ is a useful mobile node. Let $Y$ denote the common distribution of the $Y_{i}^{\prime} s$. Then $E[X(\eta, t)=(m-1) E[Y]$. To calculate $\mathrm{E}[\mathrm{Y}]$, we first condition on the direction of $M_{i}$. Observe that

$$
E\left[Y_{i}\right]=\int_{\phi=\eta-\frac{\pi}{6}}^{\eta+\frac{\pi}{6}} E\left[Y_{i} \mid d_{i}=\phi\right] \operatorname{Pr}\left[d_{i}=\phi\right]
$$

where $d_{i}$ is the direction of the node $M_{i}$.
To calculate $E\left[Y_{i} \mid d_{i}=\phi\right]$, consider the mobile node $M_{i}$ moving in direction $\phi$. Relative to $M_{0}, M_{i}$ moves at angle of $\frac{\pi}{2}+\frac{\phi}{2}$ at speed $|2||v|\left|\sin \frac{\phi}{2}\right|$.

Thus given that $d_{i}=\phi, Y_{i}=1$ iff $M_{i}$ lies in a region of area

$$
r_{0} \cdot t \cdot|2|\left|v \| \sin \frac{\phi}{2}\right|
$$

at time $t=0$. Thus,

$$
E\left[Y_{i}\right]=\frac{1}{2 \pi} \int_{\phi=\eta-\frac{\pi}{6}}^{\eta+\frac{\pi}{6}} r_{0} \cdot t \cdot|2|\left|v \| \sin \frac{\phi}{2}\right| d \phi
$$

Now $E\left[Y_{i}\right]$ is minimized when $\eta=0$ (i.e. when $M_{0}$ wishes to transmit the packet to another mobile moving in the direction of $\left.M_{0}\right)$. In this case,

$$
\begin{aligned}
E\left[Y_{i}\right] & =\frac{1}{2 \pi} \cdot r_{0} \cdot t \cdot 2\|v\| \cdot 2 \int_{0}^{\frac{\pi}{6}} \sin \frac{\phi}{2} d \phi \\
& =\frac{4 t\|v\| r_{0}}{\pi}\left(1-\cos \frac{\pi}{12}\right) \\
& >\frac{4 t\|v\| r_{0}}{\pi}(0.034)
\end{aligned}
$$

Thus $E[X(\eta, t)]>\frac{1}{24} r_{0} t\|v\| m$.

Using the second Chernoff bound (Equation 10) with $\delta=\frac{1}{2}$, we get that if $t>\frac{\log m}{\|v\| \sqrt{m}}$

$$
\operatorname{Pr}\left[X(\eta, t)<\frac{1}{2} \cdot \frac{1}{24} r_{0} t\|v\| m\right] \leq e^{-c \sqrt{\log m}}=o\left(\frac{1}{\log m}\right)
$$

where $c=\frac{1}{24} \cdot \frac{1}{8} \cdot \beta^{-\frac{1}{\alpha}} \sqrt{\frac{1}{8 \pi}}$.

In particular if a mobile node moves a distance of $\frac{\log m}{\sqrt{m}}$, it encounters at least $\Theta(\sqrt{\log m})$ other mobile nodes, with probability at least $\frac{1}{12 \log m}$ (assuming $m$ is large enough).

It follows from Lemma 6 and Lemma 7 that

Theorem 8 Let $X(t)$ denote the amount of data a mobile node $M$ transmits in time $t$, then if $t>\frac{\log m}{\sqrt{m}|| v||}$ and $n$ is sufficiently large, then

$$
\operatorname{Pr}\left[X(t)>\frac{1}{48} \cdot \frac{\beta^{-\frac{2}{\alpha}}}{8 \pi \log m} \lambda_{m m} t\right]>1-\frac{1}{3 \log m}
$$

This holds irrespective of the direction in which $M$ attempts to transmit.

Proof: For $X(t)$ to be greater than $\frac{1}{48} \cdot \frac{\beta^{-\frac{2}{\alpha}}}{8 \pi \log m} \lambda_{m m} t$, it is sufficient that the number of encounters is at least $\frac{1}{48} m r_{0} t|| v \|$ and at least half of the data transmissions are successful during these encounters. By Lemma 6 and 7, the probability of either event not holding is less than $1 / 12 \log m$ and $1 / 4 \log m$. Thus the result follows.

Having shown bounds on the amount of data that a mobile node can handoff to other nodes, in this section we show bounds on the data received by a mobile node from other mobile nodes. This will allow us to bound the probability that a packet is discarded since it could not be handoff before its deadline. 
Let $D$ denote the maximum distance between the source and the destination. Thus $D=2 / \sqrt{\pi}$. Call a packet to be in stage $k$, if the distance of the packet from the destination is between $D 3^{-\frac{k}{2}}$ and $D 3^{-\frac{k-1}{2}}$. Since the distance decreases by at least a factor of $\sqrt{3}$ after every handoff and the distance can vary from at most $2 / \sqrt{\pi}$ to at the least $1 / \sqrt{m}$. It follows easily that

Property 9 The number of different phases will be at most $\log _{\sqrt{3}} \sqrt{m}$ which is less than $\log m$.

Since each static node produces data at rate at most $\lambda(n)$, the total rate at which data is produced is $n \lambda(n)$. We say that there is an arrival into stage $k$ if a packet becomes a stage $k$ packet, and similarly there is a departure from stage $k$ if a packet becomes stage leaves stage $k$ to enter stage $k+1$ or higher. By Property 9 each packet goes through each stage at most once, thus it follows that

Property 10 The average arrival rate into each stage $k$ is at $\operatorname{most} n \lambda(n)$.

Recall that our goal is to bound the data received by a mobile node. Since there are $n$ mobile nodes we expect that each node will receive stage $k$ packets at a rate of about $n \lambda(n) / m$. However, this is not exactly true. The fact that a mobile is moving in a particular direction and is at a particular position will affect the rate at which the mobile receives packets of stage $k$. However, we can easily bound the rate by $6 n \lambda(n) / m$. The idea is that, by the nature of our algorithm, even if all the packets at a position $\vec{r}$ need to send in a particular direction $\theta$, by the nature of the algorithm they will be spread out to mobile nodes moving in a direction between $\theta-\pi / 6$ and $\theta+\pi / 6$.

We now come to the final step of the proof. Consider a packet which is in its handoff region deadline at a distance $d$. We want to bound the probability that this packet meets its deadline.

Lemma 11 Given a packet $P$ in its handoff region with a deadline at a distance $d$. Let $H(d)$ denote the amount of data that has a deadline sooner than that of P. Then, $E[H(d)]<$ $78 \lambda(n) \log n \frac{n d}{m\|v\|}$.

Proof: Let us consider a packet $P$, and let $M$ denote the mobile node which is carrying $P$. For notational convenience, suppose that $P$ enters its handoff region at time $t=0$. Let $d$ denote the distance to the deadline of $P$. Figure 3 explains the notation used in this proof. Here $A$ is the point where the handoff region begins, and $B$ is the deadline of $P$.

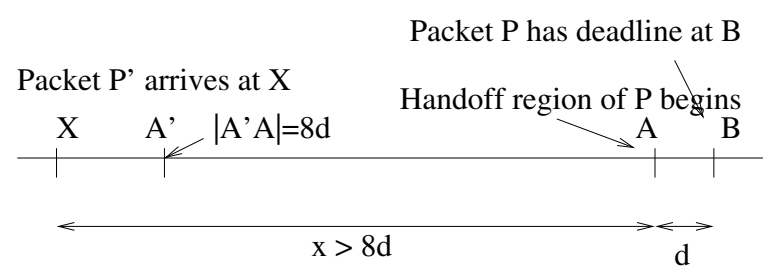

Fig. 3. Figure explaining the notation in the proof of Lemma 11

Since the handoff of packets takes place in the Earliest Deadline First (EDF) manner, the handoff of packet $P$ will be delayed by other packets carried by $M$, which have a deadline earlier than that of $P$. These packets (which have a deadline earlier than that of $P$ ) can be classified into:

1) Packets which arrive after time 0.

2) Packets which arrived before time 0 .

We will bound the delay due to each of these. It is easy to bound the delay due to packets of the first type. For the first type, the expected number of packets which arrive during the time $M$ covers a distance $d$ is bounded by $6 \lambda(n) \log m \frac{d n}{m\|v\|}$. In the worst case assume that all these packets have a deadline before the deadline of $P$, and hence the expected number of such packets which need to be handed off before $P$ will be at most $6 \lambda(n) \log m \frac{d n}{m\|v\|}$.

For the second type, we need a more detailed analysis. Consider a packet $P^{\prime}$ which arrives at a distance $x$ before $A$. This point is denoted by $X$ in Figure 3 .

We again classify these packets $\left(P^{\prime}\right)$ into two types. First we consider packets which arrive at a distance $x$ before $A$ such that $x \in[0,8 d]$. The expected number of such packets will be $8 d \log m \cdot 6 \lambda(n) n / m\|v\|$. Assuming that all these packets have a deadline in the interval $A B$ in the worst case, the expected number of packets of this type that need to handed off before $P$ will be $48 d \log m \lambda(n) n / m\|v\|$.

If $x>8 d$, we need a different argument. Observe the following:

1) If the distance of the destination of $P^{\prime}$ from $X$ is greater than $\frac{4}{3}(x+d)$, then the deadline interval of $P^{\prime}$ begins at a distance greater $(x+d)$.

2) If the distance of the destination of $P^{\prime}$ is less than $\frac{\sqrt{3}}{2} x$ then the deadline interval of $P^{\prime}$ ends at most distance $x$ from the point $X$ (hence before $A$ ).

Thus the deadline interval of $P^{\prime}$ does not intersect the segment $A B$ in either case. Thus, if the deadline interval of $P^{\prime}$ intersects $A B$, then its destination must lie between the distances $d_{\min }=$ $\frac{\sqrt{3}}{2} x$ and $d_{\max }=\frac{4}{3}(x+d)$ from $X$.

Now the ratio of the distances $d_{\max }$ to $d_{\min }$ is $8(x+$ d) $/(3 \sqrt{3} x)$ which is smaller than $\sqrt{3}$ if $x>8 d$. This implies that such a packet can lie in at most 2 phases. Thus, the expected arrival rate of such packets is at most $12 n \lambda(n) / m$. Now for any packet $P^{\prime}$, we know that it deadline is uniformly distributed in an interval of size at least $\frac{x}{4}$. Thus, the probability that $P^{\prime}$ has a deadline in the segment $A B$ is at most $\frac{4 d}{x}$. Thus the number of packets that arrive in an interval of length $d x$ will be $12 n \lambda(n) \frac{d x}{m\|v\|} \cdot \frac{4 d}{x}$.

Thus, the expected number of conflicting packets which will have deadline in the interval $A B$ will be bounded by

$$
\begin{aligned}
& 48 \frac{n \lambda(n)}{m\|v\|} \int_{x=8 d}^{\frac{2}{\sqrt{\pi}}} \frac{d}{x} d x \\
= & 48 \frac{n \lambda(n) d}{m\|v\|} \log \frac{2}{\sqrt{\pi} 8 d} \\
< & 24 n \lambda(n) \log n \frac{d}{m\|v\|} \quad\left(\text { Since } d \geq \frac{1}{\sqrt{n}}\right)
\end{aligned}
$$

Thus the expected amount of data that needs to be delivered before a packet with deadline at a distance $d$ is delivered is at $\operatorname{most} 78 n \lambda(n) \log n \frac{d}{m\|v\|}$. 
Lemma 12 There exists a constant $c>0$ such that, if $\lambda(n) \leq$ $\frac{c W m}{n \log ^{3} n}$, then the probability that a handoff is not successful is at most $\frac{1}{2 \log m}$.

Proof: Consider a packet $P$ in its handoff region which has a deadline at a distance $d$, hence (deadline approaches after time $\frac{d}{\|v\|}$. Then, this packet is discarded if and only if the data which has a deadline sooner than $d$ is more than what can be transmitted in time $t$.

From Theorem 8 , it follows that there exists a constant $c_{1}$ such that the data transmitted in time $t$ is at least $\frac{c_{1} W t}{\log m}$ with probability at least $1-\frac{1}{3 \log m}$.

Similarly by lemma 11 and the Markov Inequality, the probability that more than $(78 n \lambda(n) t \log m) / m \cdot 6 \log m$ has deadline before $P$ is at most $\frac{1}{6 \log m}$.

Choosing $c=\frac{c_{1}}{6.78}$, it follows that if $\lambda(n) \leq \frac{c W m}{n \log ^{3} m}$ the probability that the handoff is unsuccessful is less than $1 / 3 \log m+1 / 6 \log m=1 / 2 \log m$.

\section{E. Static-to-Static Phase}

Finally we note that after the mobile-to-mobile phase, the distance of a packet from the destination leader node is at most $\frac{\log m}{\sqrt{m}}$. In this stage the intermediate static leader node directly transmits the packet to the destination leader node. The coordination among the leader nodes is done as follows.

In each time slot, choose a fraction of $\frac{c^{2}}{\log ^{2} m}$ of the static nodes such that each of them are at distance of at least $\frac{\log m}{c \sqrt{m}}$ from each other. Observe that if $c$ is sufficiently small, then mimicking the proof of Theorem 3, it follows that this fraction of nodes can simultaneously transmit the packets directly to its intended destination.

Since $W / 4$ is the bandwidth available to each leader node for communication in the static-to-static phase, this constrains $\lambda(n)$ to $\Theta\left(m \frac{W}{n \log ^{2} m}\right) .^{3}$ Thus, the achievable throughput per leader node in this phase is $\Omega\left(\frac{W}{\log ^{2} m}\right)$. Since there are $O(n / m)$ static nodes per leader node, this constrains the available $\lambda(n)$ to $\Omega\left(m W / n \log ^{2} m\right)$.

\section{F. Proof of Theorem 2}

We can now prove the main result, Theorem 2.

By property 9 we know that each packet is handed off at most $\log m$ times. Also, by Lemma 12, if $\lambda(n) \leq c \frac{m W}{n \log ^{3} m}$, the probability of a successful handoff at each stage is at least $1-\frac{1}{2 \log m}$. Thus, it follows from the union bound that the probability of a packet reaching successfully from the source to the destination is at least 0.5 . Hence the achievable throughput is at least $c / 2 \cdot m W / n \log ^{3} m=\Omega\left(m W / n \log ^{3} n\right)$.

Similarly, by Theorem 3, Lemma 12 and the results in Section III-E, we observe that the transmissions during the mobileto-mobile phase put the maximum restriction of $O\left(m \frac{W}{n \log ^{3} n}\right)$ on the value of the achievable $\lambda(n)$.

Finally, from the description of the handoff algorithm in Section III-D, it follows that the distance covered by a packet during its transmission from the source to the destination is never

\footnotetext{
${ }^{3}$ A function $f(n)$ is $\Theta(g(n))$, if $\exists$ constants $c_{1}, c_{2}$ and $N$ such that $c_{1} g(n) \leq f(n) \leq c_{2} g(n), \forall n>N$.
}

more than $\frac{2 d}{v}$, where $d$ is the diameter of the network and $v$ is the velocity of the mobile nodes. Moreover, by Lemma 4 and Lemma 7, the expected duration for which a packet waits at a node trying to find another suitable mobile moving in the required direction is $O\left(\frac{d}{v} \frac{\log m}{\sqrt{m}}\right)$. Thus, as there are at most $O(\log m)$ handoffs, the total delay due to waiting is $O\left(\frac{d}{v} \frac{\log ^{2} m}{\sqrt{m}}\right)$, which is negligible compared to the delay $\frac{d}{v}$. Thus the proof of Theorem 2 follows.

\section{More General Models}

\section{A. Mobile nodes as senders and receivers}

The proof can clearly incorporate mobile nodes as senders. However, if the mobile nodes are also receivers, then since its location will not be known in general, it is impossible to do anything better than the result of [2]. However, if we make some assumptions about the model, like a mobile node has a designated static node from where it periodically collects its packets etc., then we can adapt the proof to obtain guarantees on the delay.

\section{B. Handling More General Mobility Models}

If the value of $\mu$ (the mean distance that a mobile node moves before changing its direction) is much smaller than the diameter of the network $(d)$, we can do the following two things:

1) Either modify the algorithm such that instead of sending the packet from the static source node $S$ to the static receiver node $R$ using mobile nodes, we choose intermediate nodes $S_{1}, \ldots, S_{d / \mu}$, such that we first route from $S$ to $S_{1}$, then from $S_{1}$ to $S_{2}$ and so on. This technique is also used in [4]. Note that capacity of the network becomes directly proportional to $\mu$. Hence, smaller the $\mu$, smaller the capacity.

2) Alternatively we can do the following: We handover a packet to a mobile node, either if it moves in that direction for a distance more than $\mu$ or if it moves in that direction for a distance larger than twice the distance from the destination (as in the algorithm in Section III). In the first case, we set the deadline randomly between $\mu / 2$ and $3 \mu / 4$. It can be seen that the analysis for Lemma 11 can be directly modified to handle this case. Note that the node moves distance of at least $\mu / 2$ in this way, and we can also find a constant fraction $(>1 / e)$ of mobile nodes which satisfy this property. Thus there are at most $1 / \mu$ such steps. In the second case, the analysis remains the same as that in Section III and there are $\log n$ such steps. Thus we obtain a throughput of the order of $W m /\left(n \log ^{2} n\left(\log n+\frac{1}{\mu}\right)\right)$.

Finally, we note that our results are easily extended to the following:

1) The results still hold (up to a multiplicative constant) for models where the steady state distribution of the static nodes is some homogeneous distribution rather than the special case of the uniform distribution. Here, a homogeneous distribution refers to a distribution in which the probability distribution function lies between constants $c_{\min }$ and $c_{\max }$. 
2) Similarly, the speeds of mobile nodes need not be identical. If they are upper and lower bounded by $v_{\max }$ and $v_{\text {min }}$, respectively, the analyses go through except for different constants.

3) The results can also be rederived for a three dimensional setting. Recently, results for 3-d settings in other models have been obtained [3], [21].

\section{Discussions}

In this section we discuss the implications of the results obtained in the previous sections. We observe the following:

1) The delay experienced by a packet is inversely proportional to the speed of the mobile nodes.

2) The throughput $\lambda(n)$ obtained is independent of the velocity of the mobile nodes.

3) In this paper, we only derive bounds on $\lambda(n)$ which ensure a packet loss probability of at most 0.5 . This happens since we bound the loss probability at each handoff by $1 / 2 \log m$. Note that the loss takes place due to two things: First, loss due to the wireless communication. To handle this we can reduce the value of $x$ in Lemma 5 (i.e. two nodes communicate only when they are extremely close). Second, the loss also occurs when a mobile node cannot hand off all its packets by their deadlines to appropriate mobile nodes and hence has to discard them. We can handle this by choosing a larger constant in the application of Markov's Inequality in Lemma 12. This reduces the value of $\lambda(n)$ by the same constant (i.e. if $p$ is the loss probability, then $\lambda(n) \propto p)$.

4) We can obtain bounds on the expected buffer occupancy at the mobile nodes. In particular, applying Little's Law [10] to Theorem 2 we get the expected amount of data in the buffers is bounded by $\frac{2 d}{v} \frac{W}{\log ^{3} n}$.

\section{CONCLUSIONS}

In this paper we have proposed a routing algorithm for adhoc networks with the goal of achieving close-to optimal capacity while keeping the delay small. Our algorithm exploits the patterns in the mobility of nodes to provide guarantees on the delay. Moreover, the throughput achieved by the algorithm is only a poly-logarithmic factor off from the optimal.

It is a challenging problem to define a model which is general enough to capture a wide range of mobility patterns of users and at the same time is also analytically tractable. In this paper we have taken a modest step towards incorporating and considering the effects of mobility patterns of nodes in the analysis of ad hoc routing protocols. We developed algorithmic ideas to bound and analyze the delay, these could be of independent interest.

While there are many directions in which the model can be extended and many other issues that need to be considered in analyzing the performance of ad hoc networks, we believe that our work could provide intuition for future protocol designs and for rigorous analysis in ad hoc networks. While we focus only on the network capacity and the delay, it would be interesting to consider other metrics like power consumption, the number of hops to route the packet, fault tolerance, minimizing the number of control packets etc. or a combination thereof.

\section{REFERENCES}

[1] W. Feller. An Introduction to Probability Theory and its applications, volume 2. John Wiley \& Sons, 2 edition, 1971.

[2] Mathias Grossglauser and David Tse. Mobility increases the capacity of ad-hoc wireless networks. In Proceedings of IEEE Infocom'01, April 2001.

[3] P. Gupta and P. Kumar. Internets in the sky: The capacity of three dimensional wireless networks. Communications in Information and Systems, 1:39-49, 2001.

[4] Piyush Gupta and P. R. Kumar. The capacity of wireless networks. IEEE Transactions on Information Theory, 46(2):388-404, 2000.

[5] B. Hajek, A. Krishna, and R. LaMaire. the capture probability for a large number of stations. IEEE Transactions on Communications, 45(2):254 260, February 1997.

[6] X. Hong, M. Gerla, G. Pei, and C. Chiang. A group mobility model for ad hoc wireless networks, 1999.

[7] David B Johnson and David A Maltz. Dynamic source routing in ad hoc wireless networks. In Mobile Computing, volume 353. Kluwer Academic Publishers, 1996.

[8] Jinyang Li, John Jannotti, Douglas S. J. De Couto, David R. Karger, and Robert Morris. A scalable location service for geographic ad hoc routing. In ACM Mobicom, 2000.

[9] B. Liang and Z. Haas. Predictive distance-based mobility management for pcs networks. In Proceedings of IEEE Infocom'99, 1999.

[10] J. Little. A proof of the queueing formula $l=\lambda w$. Operations Research, 9:383-387, 1961.

[11] R. Motwani and P. Raghavan. Randomized Algorithms. Cambridge University Press, 1995.

[12] Shree Murthy and J.J. Garcia-Luna-Aceves. An efficient routing protocol for wireless networks. Mobile Networks and Applications, 1:183-197, 1996.

[13] Vincent D. Park and M. Scott Corson. A highly adaptive distributed routing algorithm for mobile wireless networks. In Proc. IEEE Infocom, 1997.

[14] Charles E. Perkins and Elizabeth M. Royer. Ad-hoc on-demand distance vector routing. In Proc. of the $2^{\text {nd }}$ IEEE Workshop on Mobile Computing Systems and Applications, pages 90-100, 1999.

[15] Ivan Stojmenovic Prosenjit Bose, Pat Morin and Jorge Urrutia. Routing with guaranteed delivery in ad hoc wireless networks. In Proc. of the $3^{\text {rd }}$ International Workshop on Discrete Algorithms and Methods for Mobile Computing and Communications, pages 48-55, 1999.

[16] Ram Ramanathan and Regina Rosales-Hain. Topology control of multihop wireless networks using transmit power adjustment. In Proc. IEEE Infocom, pages 404-413, 2000.

[17] Volkan Rodoplu and Teresa H. Meng. Minimum energy mobile wireless networks. In Proc. IEEE Int. Conf. on Communications, pages 1633 1639, 1998.

[18] Paramvir Bahl Roger Wattenhofer, Li Li and Yi-Min Wang. Distributed topology control for power efficient operation in multihop wireless ad hoc networks. In Proc. IEEE Infocom, 2001.

[19] Ivan Stojmenovic and Xu Lin. Power-aware localized routing in wireless networks. In Proc. IEEE Int. Parallel and Distributed Processing Symp., 2000.

[20] Mike Woo Suresh Singh and C. S. Raghavendra. Power-aware routing in mobile ad hoc networks. In Proc. ACM/IEEE Int. Conf. on Mobile computing and networking, pages 181-190, 1998.

[21] S. Toumpis and A. J. Goldsmith. Ad hoc network capacity. In Asilomar Conference of Signals, Systems and Computers, 2000.

[22] H. Xie and D. Goodman. Mobility models and biased sampling problem. In IEEE ICUPC, volume 2, pages 803-807, 1993.

\section{APPENDIX}

\section{A. Chernoff Bounds}

Chernoff Bounds[11]: Let $X_{1}, \ldots, X_{n}$ be independent 0-1 random variables such that $\operatorname{Pr}\left[X_{i}=1\right]=p_{i}$. Let $X=X_{1}+$ $\ldots+X_{n}$ and $\mu=E[X]$. Then,

$$
\begin{gathered}
\forall \delta>0, \quad P[X>(1+\delta) \mu]<\left[\frac{e^{\delta}}{(1+\delta)^{1+\delta}}\right]^{\mu} \\
\forall \delta \in[0,1], \quad \operatorname{Pr}[X<(1-\delta) \mu]<e^{-\frac{\mu \delta^{2}}{2}}
\end{gathered}
$$




\section{B. Proof of Theorem 1}

Proof: Let us first consider a discrete version of the process. Assume that the time is slotted and events occur at time $t_{0}, 2 t_{0}, 3 t_{0}, \ldots$. At any time step, the node might change its direction with probability $p=\frac{v t_{0}}{\mu}$, or else it continues in the current direction with probability $1-p$. Observe the distance covered by the walk before switching directions is a geometrically distributed random variable with mean $\mu$. As $t_{0} \rightarrow 0$, this discrete model approaches the continuous model.

Part 1 of the theorem follows from the fact that the motion of the $n$ nodes is uncorrelated to each other. To show the second and third parts, we will use the balance equations and show that the uniform distribution satisfies them.

Let $\pi(\vec{r}, \theta)$ denote the steady state probability of being in an infinitesimal disk of area $d A$ around position $\vec{r}$ and the direction of motion being between angles $\theta$ and $\theta+d \theta$.

Let $\vec{r}$ be a point sufficiently in the interior of the disk. Then, since the walk leaves the point $\vec{r}$ and moves to $\vec{r}+|v| t_{0} \hat{u}(\theta)$, where $\hat{u}(\theta)$ is a unit vector in the direction $\theta$. The rate of leaving the disk will be $\pi(\vec{r}, \theta)$.

To calculate the rate of entering the disk, observe that the probability of entering the disk will be made up of two components. The first component is the probability that the node was at position $\vec{r}-|v| t_{0} \hat{u}(\theta)$ at the previous time step and that it did not change direction. And the second component is that the node changed direction on reaching $\vec{r}$.
This gives us that the rate of entering the disk will be:

$$
(1-p) \pi\left(\vec{r}-|v| t_{0} \hat{u}(\theta), \theta\right)+p \frac{1}{2 \pi} \int_{\theta^{\prime}=0}^{2 \pi} \pi\left(\vec{r}-|v| t_{0} \hat{u}\left(\theta^{\prime}\right), \theta^{\prime}\right) d \theta^{\prime}
$$

Thus the rate balance equation gives

$$
\begin{aligned}
\pi(\vec{r}, \theta)= & (1-p) \pi\left(\vec{r}-|v| t_{0} \hat{u}(\theta), \theta\right) \\
& +p \frac{1}{2 \pi} \int_{\theta^{\prime}=0}^{2 \pi} \pi\left(\vec{r}-|v| t_{0} \hat{u}\left(\theta^{\prime}\right), \theta^{\prime}\right) d \theta^{\prime}
\end{aligned}
$$

Observe that setting all $\pi(\vec{r}, \theta)$ to be the same in the interior of the disk satisfies the balance equation. It can be shown that all solutions to the above balance equation are uniform distributions. Indeed, if $\pi(\vec{r}, \theta)$ is not a constant, then when $\pi(\vec{r}, \theta)$ is a local maximum, the equality in (11) no longer holds.

Now, consider the behavior on the disk edge. Let the state of the walk be $(\vec{r}, \theta)$, and $\vec{r}$ be on the edge of the disk, such that $\vec{r}+$ $|v| t_{0} \hat{u}(\theta)$ lies outside the disk. The behavior of the walk is the following: The walk does not change direction with probability $1-p$, in this case it is reflected at the boundary at reaches a position $\left(\overrightarrow{r^{\prime}}, \bar{\theta}\right)$ where $\overrightarrow{r^{\prime}}$ and $\bar{\theta}$ are the location and the direction obtained by reflecting the walk. Or, with probability $p$ the walk chooses a direction $\theta^{\prime}$ uniformly at random from $[0,2 \pi)$, if the direction $\theta^{\prime}$ points outside the disk, then the walk remains in state $(\vec{r}, \theta)$, else it moves to the state $\left(\vec{r}+|v| t_{0} \hat{u}\left(\theta^{\prime}\right), \theta^{\prime}\right)$. Again, it is seen that setting all $(\pi(\vec{r}, \theta) i)$ to be the same satisfies the balance equations. Finally, as $t_{0}$, hence $p$, gets arbitrarily small this corresponds to the description of the walk in the uniform mobility model. Thus the result follows. 